\section{Formación científica en el pregrado de medicina en Chile: ¿dónde estamos? y ¿hacia dónde vamos?}

\author{
FRANCISCO GARRIDO C. ${ }^{1,2}$, TOMAS P. LABBÉ ${ }^{3}$, \\ ENRIQUE PARIS M. ${ }^{4}$, JUVENAL A. RÍOS ${ }^{5,6}$,
}

\section{An analysis of scientific formation in medical schools}

For more than a century the training of medical professionals has been organized according to the Flexnerian model, which comprises three cycles: basic, clinical and clerkship. On the other hand, the accelerated development of biomedical sciences modified the competences of the first cycle. Additionally, new skills required for medical practice, such as teamwork and innovation as a tool to solve health problems, challenged in recent years the classic paradigm of medical education. Therefore, the medical schools have developed multiple strategies to deal with it, such as curricular integration using competency-based education models, incorporating basic and clinical sciences in parallel during the curriculum, ensuring a relevant and applicable scientific knowledge throughout the training process. Although in Chile the Flexner prototype is still followed, the basic sciences are taught as single or integrated courses or using a systems approach. In this article we report a diagnosis about the local integration of fundamental sciences in medical training. We also compare our schools with those of Canada, Europe and Latin America. Recommendations aimed at modernizing medical school curricula are made.

(Rev Med Chile 2020; 148: 1011-1017)

Key words: Competency-Based Education; Curriculum; Natural Science Disciplines; Education, Medical, Undergraduate; Interdisciplinary Studies.

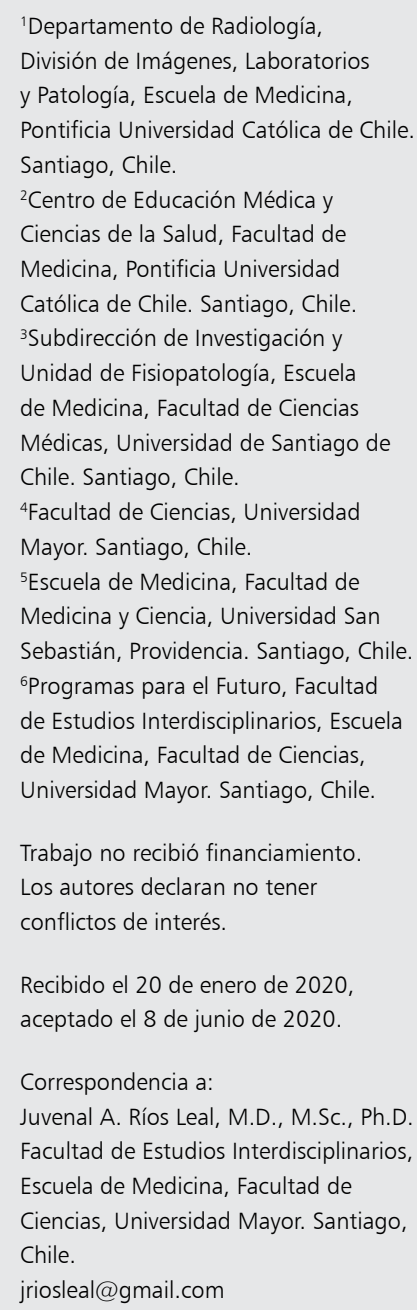

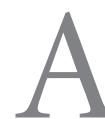
principios del siglo XX, el reconocido educador norteamericano Abraham Flexner sentó las bases de la educación médica del futuro, tras la publicación en 1910 de "Medical Education in the United States and Canada: A report to the Carnegie Foundation for the Advancement of Teaching, Bulletin No. 4", también conocido como Informe Flexner. Tras visitar 155 escuelas de Medicina de Estados Unidos de Norteamérica y Canadá, Flexner sirvió de articulador entre la entonces desprestigiada educación médica norteamericana y el sistema de salud de la época, estimulando el inicio de una importante reforma ${ }^{1}$. Influenciado por sus estudios en Alemania, Flexner identificó el currículum de la escuela de Medicina de The Johns Hopkins University como el estándar contra el cual comparó todas las escuelas visitadas. Las recomendaciones de Flexner, como el establecimiento de altos estándares de calidad y rigor en los procesos educativos, influenciaron la profesionalización y estandarización de la educación médica y continúan vigentes en la estructura de la escuela de Medicina actual ${ }^{2}$. En su informe, Flexner sugirió un curriculum médico estructurado en dos años de ciencias básicas, seguido de dos años de experiencias clínicas $(2+2)$. La adopción de esta estructura se basa en que las ciencias juegan un rol central en la práctica de la Medicina, por lo que 
el médico debe "adquirir los métodos, estándares y hábitos de la ciencia”, diferenciándolo de la formación de un técnico profesional ${ }^{1-3}$.

En la década de 1970-79, la literatura educacional reporta las primeras luces de un nuevo paradigma de educación médica basada en competencias, en que los profesionales debían prepararse para practicar la medicina en un nivel esperado de capacidades, "de acuerdo con las condiciones locales, para satisfacer las necesidades locales"4. Como definición, la educación basada en competencias es un enfoque en el cual el currículo se construye a partir de las características que el estudiante debe demostrar al final del proceso educativo, es decir, el producto final esperado es el que guía la construcción del plan de estudios ${ }^{5}$. Existen perfiles de competencias de educación médica de pregrado que sirven como marco de referencia para la implementación de este paradigma en la práctica, como "The Scottish Doctor" o "Tomorrow's Doctor", los que incorporan dentro de las competencias del egresado una formación sólida en ciencias básicas que permitan reconocer, explicar y manejar los problemas de salud usando los principios del conocimiento científico actual y los principios que sustentan la medicina ${ }^{6}$.

El desarrollo de todas las áreas de la Medicina en las últimas décadas ha traído como consecuencia una necesaria reforma de los planes de estudio de pregrado. Lo anterior, sumado a los cambios culturales, legales y sanitarios obliga a replantear los procesos para formar nuevos facultativos, con el propósito de determinar si los egresados actuales se encuentran preparados para enfrentar la práctica médica del mañana. Este análisis ha llevado a la incorporación de nuevos tópicos que desarrollen habilidades comunicativas, gestión, liderazgo y trabajo en equipo o innovación, en desmedro de otros contenidos tradicionales, como las ciencias morfológicas, que han experimentado una fuerte disminución de creditaje ${ }^{7-9}$. Mención aparte merece el importante rol que las ciencias sociales, humanidades médicas y la ética tienen en la formación médica y su contribución a la comprensión de la experiencia humana de salud y enfermedad en su complejidad, diversidad e imprevisibilidad, sobre todo en la práctica de la Medicina en el primer nivel de atención, donde el diagnóstico biológico en un contexto biográfico y social pone al médico la oportunidad de aplicar las ciencias básicas y clínicas en el marco de una "medicina humanista"10.
Es innegable el aporte de las ciencias básicas en la formación médica de pregrado. El profesional médico requiere un entendimiento profundo de la condición humana normal y patológica que le permitan realizar un diagnóstico certero y plantear opciones terapéuticas acordes para el paciente en particular'.

Merecen analizarse algunas áreas en donde una robusta formación del ciclo básico/preclínico (bioquímica, biología molecular, ciencias morfológicas, fisiología, fisiopatología y farmacología), influye e impacta en un eficiente desempeño del médico clínico de hoy y del futuro:

- Biología molecular: Su estudio adquiere especial relevancia con todo el conocimiento generado tras la secuenciación del genoma humano ${ }^{11,12}$. Cada vez más los médicos, especialistas o no, estarán expuestos a interpretar determinaciones moleculares que al menos direccionan conductas clínicas, desde una oportuna derivación, consejería genética, hasta indicación de terapias biológicas dirigidas, paradigma central de la medicina personalizada de precisión ${ }^{13-15}$. - Semiología: Los estudiantes que cuentan con conocimientos sólidos en el porqué de los signos y síntomas de una patología en particular tendrán una mejor retención de los síndromes con el paso del tiempo, dado que recurrirán a la lógica asimilada en fisiología y fisiopatología ${ }^{16}$. - Farmacología: En palabras del Dr. Jorge Mardones Restat, "es una disciplina en donde se interceptan las ciencias básicas con la práctica clínica"17. Asimismo, reportes en Estados Unidos de Norteamérica (EE.UU.) muestran que la prescripción de fármacos origina alrededor de 7.000 muertes al año ${ }^{18}$. Así, esta disciplina influye directamente en una cuestión que puede ser de vida o muerte. Su estudio obliga a enseñar desde las cuestiones mecanísticas (farmacodinámica) hasta principios clínicos como la posología, interacciones farmacológicas, bioequivalencia y la biosimilaridad, entre otras $^{19}$.

\section{Situación internacional}

\section{Canadá}

La Asociación de Facultades de Medicina, en dos de sus informes ejecutivos, analiza la formación científica en pregrado de Medicina. En uno entrega una serie de recomendaciones 
para fortalecer este ámbito ${ }^{20} \mathrm{y}$ en el otro relatan las experiencias individuales que cada Escuela de Medicina ha implementado en este sentido ${ }^{21}$. Se propone (i) involucrar a científicos básicos, junto a los clínicos y educadores médicos, en el diseño del currículum educativo de los médicos; (ii) reducir las barreras departamentales dentro de las facultades para permitir la integración óptima de los servicios clínicos con ciencias básicas y (iii) apoyar programas existentes y nuevos que capacitan en investigación a los residentes y especialistas. Por otra parte, se reportan las principales medidas que la mayoría de las escuelas han adoptado, entre ellas destacan: (i) los currículum integrados enfocados en la resolución de problemas clínicos utilizando ciencias básicas; (ii) la incorporación de un proyecto de investigación entre $1^{\circ}$ y $4^{\circ}$ año; (iii) la introducción opcional de doble titulación vía $\mathrm{MD} / \mathrm{MSc}$ o $\mathrm{MD} / \mathrm{PhD}$, adicionando 1 o 2 años más al plan de estudios regular y (iv) un examen global al término del ciclo básico como requisito para acceder al ciclo clínico.

\section{Europa}

En 1999, el Área de Educación Superior Europea (EHEA), en el llamado Proceso de Bologna (48 países) establecieron las bases para regular varias carreras de la salud en un proceso de dos ciclos separados $(3+3)$, similar al modelo británico con una lógica de obtención de bachillerato y luego la maestría sin integración de contenidos. Esta estructura fue adoptada tan solo por 7 países ${ }^{22}$. Más tarde (2010), la Asociación Europea de Escuelas de Medicina y algunos de sus más connotados educadores médicos (Allan Cumming, Josef Pfeilschifter) han ido redefiniendo un modelo integrado de dos ciclos $\left(3+3^{\star}\right)$ en un "continuum" de contenidos básicos y clínicos que se inician al mismo tiempo, pero que van variando su representación (importancia) porcentual a lo largo del currículo ${ }^{23}$. Uno de los pocos países europeos que mantiene una estructura más "clásica" con un claro ciclo básico (preclínico) de dos años y a continuación 4 netamente clínicos $(2+4)$ es Alemania $^{24}$.

\section{Latinoamérica}

El trabajo de Pernas y col (2012) ${ }^{25}$ recopila las experiencias a las cuales se dio seguimiento tras la reforma de 2007 de las Escuelas de Medicina de Cuba. En este reporte se hace una descripción histórica del proceso, se releva la figura del profesor Fidel Ilizástigui como educador médico, quien ya en 1990 proponía la integración del ciclo de ciencias básicas biomédicas (CBB) con la enseñanza de la medicina general integral (MGI). La estrategia apuntaba a que los profesores clínicos iban solicitando permanentemente contenidos necesarios a las CBB para el desarrollo de destrezas del ciclo de MGI. En este sentido, el plan de acción consistía en incrementar el número de discusiones de casos, las reuniones clínico-patológicas y clínico-radiológicas.

\section{Situación nacional}

En nuestro país, tanto las competencias comunes definidas para los egresados de las Escuelas de Medicina de la Asociación de Facultades de Medicina de Chile (ASOFAMECH) como el perfil de conocimientos del Examen Único Nacional de Conocimientos en Medicina (EUNACOM) no hacen referencia explícita a los contenidos de ciencias básicas necesarias para las formación médica ${ }^{26,27}$. Sin embargo, ante los nuevos desafíos, las distintas escuelas de Medicina del país han mostrado en la última década un rediseño de sus mallas para el primer ciclo, transitando desde los cursos monodisciplinares a asignaturas que evidencian grados variables de integración entre disciplinas.

En la Tabla 1 se resumen datos sobre la estrategia de enseñanza-aprendizaje de las ciencias fundamentales de las 20 escuelas de Medicina que integran el directorio de ASOFAMECH. A través de un análisis preliminar es posible apreciar al menos cuatro estructuras o estrategias de diseño curricular para el ciclo formativo que estamos analizando: Grupo i; aquellas que han adoptado el sistema de asignaturas integradas por disciplinas afines, como por ejemplo: bases morfológicas de la Medicina (anatomía, histología y embriología), bases funcionales de la Medicina (biología molecular, bioquímica, fisiología y fisiopatología) o el estudio de los agentes patógenos de la enfermedad (microbiología, parasitología e inmunología); Grupo ii; aquellas que han preferido mantener o bien han regresado a las asignaturas monodisciplinares separadas como "cátedras" claramente individualizables; Grupo iii; aquellas carreras que presentan una estrategia mixta, entre los asignaturas integradas y algunas disciplinas que se siguen abordando de manera individual, en estas 
Tabla 1. Organización curricular del ciclo básico de las Escuelas de Medicina pertenecientes a ASOFAMECH

\begin{tabular}{|c|c|c|}
\hline Tipo de currículo en el ciclo básico & n de Escuelas & Escuela \\
\hline Predominantemente integrado (i) & 2 & $\begin{array}{l}\text { Universidad Diego Portales } \\
\text { Universidad de la Frontera }\end{array}$ \\
\hline Predominantemente monodisciplinar (ii) & 11 & $\begin{array}{l}\text { Universidad de Antofagasta } \\
\text { Universidad de Chile } \\
\text { Universidad de Concepción } \\
\text { Universidad de los Andes } \\
\text { Universidad Finis Terrae } \\
\text { Universidad Mayor } \\
\text { Universidad Andrés Bello } \\
\text { Universidad de Santiago de Chile } \\
\text { Universidad Católica del Norte } \\
\text { Universidad Católica de la Santísima Concepción } \\
\text { Universidad de Talca }\end{array}$ \\
\hline Mixto (iii) & 6 & $\begin{array}{l}\text { Universidad San Sebastián } \\
\text { Universidad del Desarrollo } \\
\text { Pontificia Universidad Católica de Chile } \\
\text { Universidad Austral de Chile } \\
\text { Universidad Autónoma de Chile } \\
\text { Universidad Católica del Maule }\end{array}$ \\
\hline Por sistema (iv) & 1 & Universidad de Valparaíso \\
\hline
\end{tabular}

excepciones destacan los ramos de neurociencias, genética médica, farmacología o fisiopatología como aquellas más frecuentemente impartidas de manera individual en el formato "cátedra" y el Grupo iv; aquellas que imparten todas las disciplinas antes mencionadas bajo una organización anatomo-fisiológica en sistemas (cardiovascular, locomotor, digestivo, etc.).

\section{Recomendaciones}

\section{A nivel de la escuela de Medicina}

Con el propósito de otorgar la relevancia de las ciencias básicas en la formación médica, la literatura reporta diferentes modelos de integración curricular que utilizan el tiempo y las disciplinas clínicas y científicas como puntos de referencia. Entre ellas se encuentran la integración horizontal, vertical y en espiral ${ }^{28}$. La integración horizontal propone la articulación de disciplinas en un período finito de tiempo, como el aprendizaje de ciencias básicas afines en cursos unificados (ejemplo: morfología humana, integrando anatomía, embriología e histología). Por otro lado, la integración vertical representa la articulación de las disciplinas a lo largo del tiempo, rompiendo las barreras entre las ciencias básicas y clínicas. Dentro de este enfoque encontramos el "modelo Z”, el cual permite una introducción precoz a la clínica, mientras se mantiene un componente de ciencias básicas a lo largo del currículo (Figura 1). Finalmente, en el modelo de integración curricular en espiral de Dundee, que combina las ventajas de los dos anteriores, las ciencias básicas y clínicas interactúan entre sí en todas las etapas del currículo, permitiendo el tránsito de los estudiantes a partir del estudio de la estructura y función normal hasta llegar al aprendizaje en la práctica (Figura 1$)^{28}$.

En cuanto a estrategias metodológicas, a través de casos clínicos el aprendizaje basado en problemas se reconoce como un facilitador del encuentro entre las ciencias básicas y clínicas. Sin embargo, la profundidad de los aprendizajes no es uniforme y depende en gran parte de la motivación del propio estudiante ${ }^{29}$. Existen tópicos básicos que serán relevantes en el contexto clínico, los cuales son susceptibles de trasladar a los cursos superiores. Por ejemplo, la anatomía y fisiología de los órganos de los sentidos podrían ser tratados en los cursos de las especialidades correspondientes, 


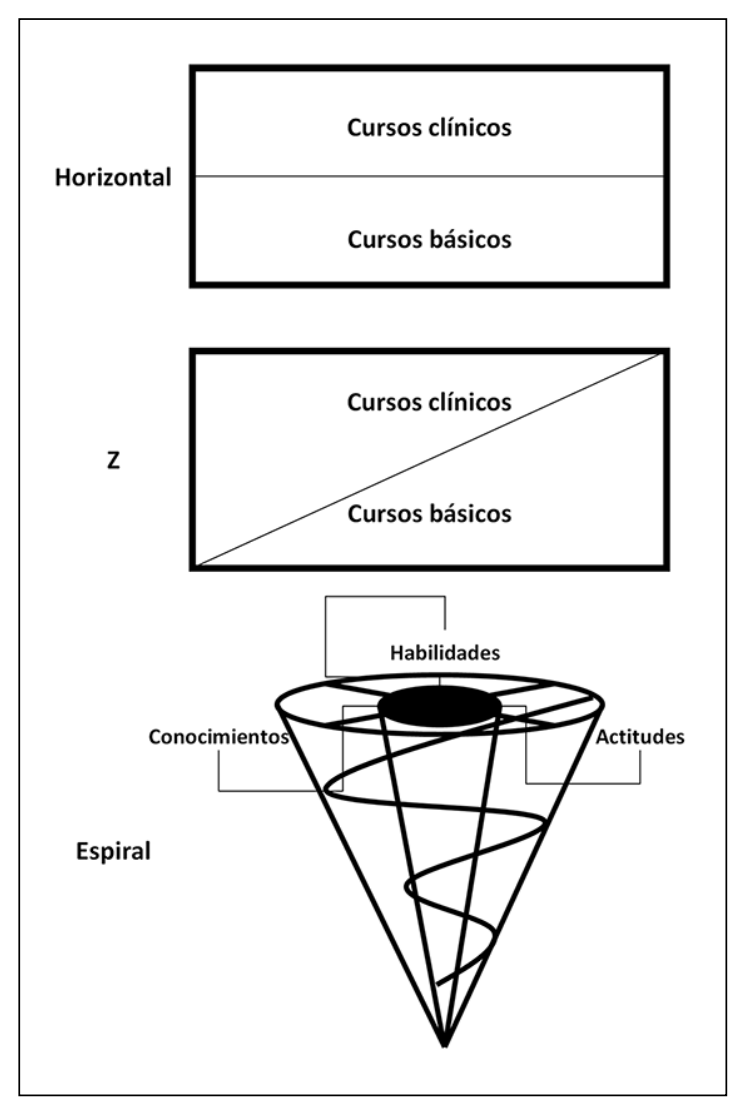

Figura 1. Modelos de estructuras curriculares en Medicina. Leyenda: Horizontal (arriba), Z (al medio) y espiral (abajo).

lo mismo pasa con farmacología. Así es posible fomentar la participación de los docentes básicos en el segundo ciclo, lo que no es muy frecuente.

\section{A nivel de la Universidad}

En cuanto al desarrollo organizacional, existen aún barreras que no permiten la fluida interacción entre los docentes básicos y clínicos, ya se ha explicado que está dado por la organización física de las instituciones (paradigma universidad y hospital), motivaciones académicas diferentes o la compartimentalización de las facultades, entre otros. Una estrategia para acercar a estos docentes es la incorporación de investigadores básicos a los departamentos clínicos, que permitan el desarrollo de investigación aplicada, sirviendo de modelo para los estudiantes y el resto de la comunidad académica. Por otro lado, existe amplio debate sobre la implementación de programas de doble titulación $\mathrm{MD} / \mathrm{PhD}$, los cuales ya existen a nivel internacional y que permiten el entrenamiento de investigadores con orientación básica y clínica que renueven la planta académica de las facultades de Medicina ${ }^{30}$.

\section{Conclusiones y desafíos}

Los lineamientos propuestos en la Figura 2 pueden sentar las bases de una agenda modernizadora del ciclo básico de las escuelas de Medicina del futuro, asimismo, un desafío pendiente en el medio local es la realización de análisis y estudios periódicos, que permitan evaluar sistemáticamente el real impacto de estas "reformas" en la educación médica chilena. Sin embargo, es necesario precisar algunas situaciones que deben suscitarse e impulsarse en corto y mediano plazo, y que sin ellas sería imposible dar sostenibilidad a una malla curricular actualizada y acorde a los desafíos que implica estar en sintonía con los permanentes avances científicos y tecnológicos. Se considera central para este proceso transformador:

i. Cuidar que la masa crítica de docentes en algunas disciplinas "clásicas" no pierda vigor $y$ vigencia en el tiempo, tal es el caso de los anatomistas, histólogos, embriólogos y probablemente parasitólogos. Activar o reactivar programas de Magíster vinculados a estas áreas, de forma paralela a especialidades médicas parece una alternativa plausible para la mantención del capital humano docente.

ii. Si bien el objetivo de nuestras escuelas no es egresar médicos-científicos como tales, el tener docentes científicos con formación médica permite hacer las relaciones correctas que el estudiante requiere para dar sentido clínico a los tópicos revisados en el ciclo básico ${ }^{31}$. En este sentido, varios autores locales e internacionales, incluyendo ganadores del Premio Nobel, han advertido el preocupante retroceso que está teniendo esta "especie" en el ecosistema sanitario ${ }^{32-34}$.

iii. Pensar "fuera de la caja" cuando se diseñen e implementen procesos de reforma curricular es abrirse a la posibilidad de nuevas asignaturas. Estas podrán ser fundamentales para abordar los desafíos de la Medicina del futuro, como es el caso de la salud digital, inteligencia artificial, medicina genómica, medicina personalizada de precisión, medicina traslacional, nutrigenómica y medicina regenerativa, entre otras. 


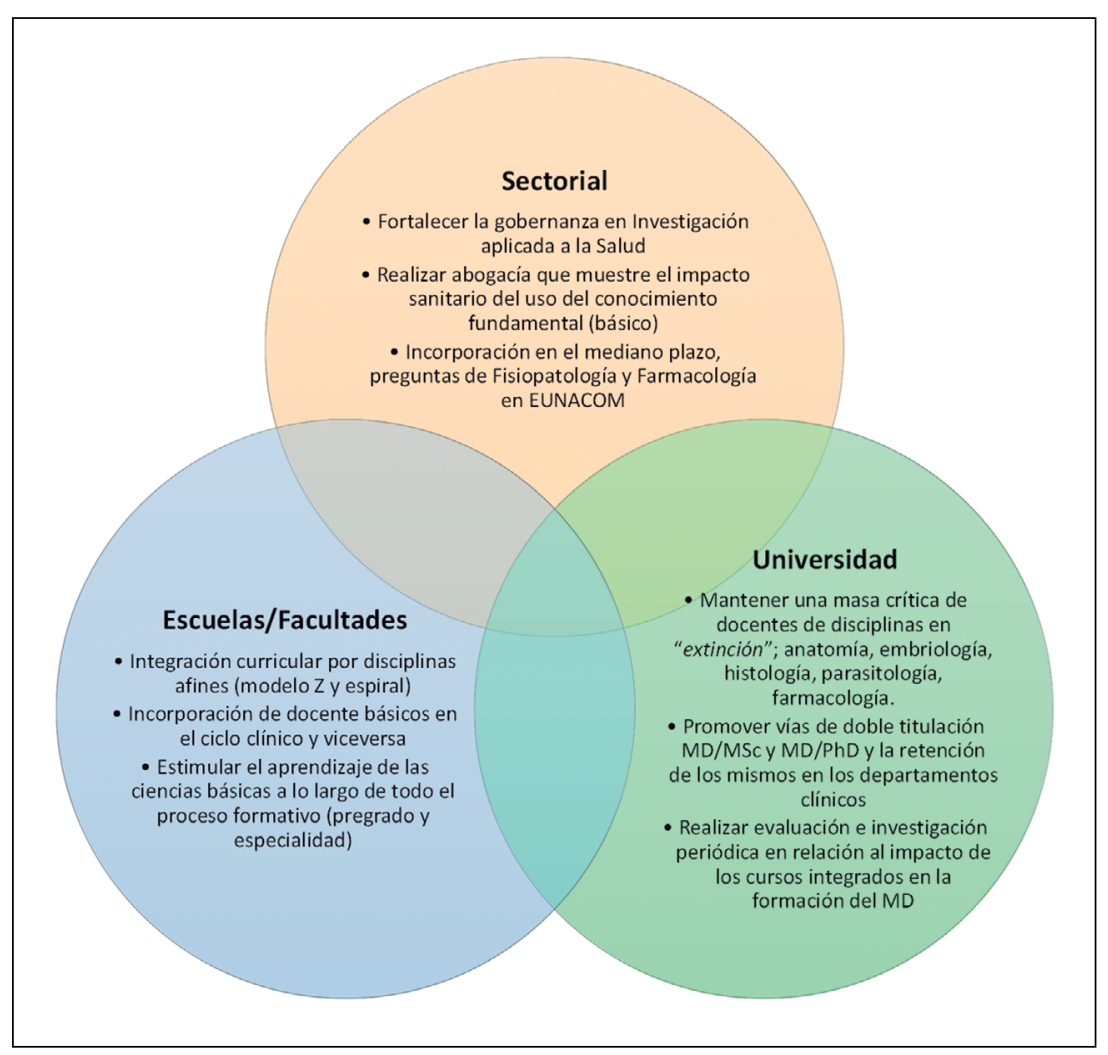

Figura 2. Resumen de recomendaciones para modernizar el ciclo básico. Leyenda: Algunas de estas recomendaciones, transitan en el límite de iniciativas sectoriales (Estado), universidad y el microambiente de las facultades/escuelas.
Una adecuada formación científica durante el pregrado es clave por su contribución al desarrollo del pensamiento crítico y para sentar las bases del razonamiento clínico ${ }^{35}$. Es por ello, por lo que, al revisar la experiencia internacional, nos encontramos ubicuamente con que este ciclo formativo va en estrecha relación a la investigación como parte del entrenamiento médico. En palabras de Santiago Ramón y Cajal el imperativo es: "Inculcadles a nuestros hijos los métodos de estudio, los principios luminosos a cuya aplicación se deben los descubrimientos científicos. Cread no eruditos y quietistas, sino voluntades enérgicas, espíritus reformadores para llevar la idea a la realidad".

Agradecimientos: Los autores agradecen muy espacialmente a las Escuelas de Medicina donde se desempeñan como docentes y autoridades, según corresponda; Pontificia Universidad Católica de Chile (FJG), Universidad de Santiago de Chile (TPL), Universidad Mayor (EPM y JAR) y Universidad San Sebastián (JAR y TPL).

\section{Referencias}

1. Barzansky B. Abraham Flexner and the Era of Medical Education Reform. Acad Med 2010; 85 (9 Suppl): S1925.

2. Finnerty EP, Chauvin S, Bonaminio G, Andrews M, Carroll RG, Pangaro LN. Flexner revisited: the role and value of the basic sciences in medical education. Acad Med 2010; 85 (2): 349-55.

3. Sibbald $\mathrm{M}$, Neville A. A hundred years of basic science in medical education. Perspect Med Educ 2016; 5 (3): 136-7.

4. Powell DE, Carraccio C. Toward Competency-Based Medical Education. N Engl J Med 2018; 378 (1): 3-5.

5. Harden RM, Crosby JR, Davis MH, Su MM, Ar Y. AMEE Guide No. 14: Outcome-based education: Part 1 An introduction to outcome-based education. Med Teach 1999; 21 (1): 7-14.

6. Simpson JG, Furnace J, Crosby J, Cumming AD, Evans PA, Friedman Ben David M, et al. The Scottish doctor-learning outcomes for the medical undergraduate in Scotland: a foundation for competent and reflective 
practitioners. Med Teach 2002; 24 (2): 136-43.

7. Garrido F, Burdiles Á, Arau R, Cisternas M. Desarrollo de un currículum de Radiología para la formación médica de pregrado: Experiencia de una Escuela de Medicina de Chile. Rev Chil Radiol 2018; 24 (3): 87-93.

8. Inzunza $\mathrm{O}$, Vargas $\mathrm{A}, \mathrm{Bravo} \mathrm{H}$. Anatomy, and neuroanatomy the most impair in the curricular reform. Int J Morphol 2007; 25 (4): 825-30.

9. Weston WW. Do we pay enough attention to science in medical education? Can Med Educ J 2018; 9 (3): e10914.

10. Evans M. Medicine, philosophy, and the medical humanities. Br J Gen Pract 2002; 52 (479): 447-9.

11. Collins FS, McKusick VA. Implications of the human genome project for medical science. J Am Med Assoc 2001; 285 (5): 540-4.

12. Hood L, Rowen L. The human genome project: big science transforms biology and medicine. Genome Med 2013; 5 (9): 79.

13. Kumar D. Clinical molecular medicine: principles and practice. $1^{\text {st }}$ Edition. Academic Press. 2019.

14. Khatry DB. Precision medicine in clinical practice. Per Med 2018; 15 (5): 413-7.

15. Chow N, Gallo L, Busse JW. Evidence-based medicine and precision medicine: Complementary approaches to clinical decision-making. Precis Clin Med 2018; 1 (2): 60-4.

16. Woods NN, Brooks LR, Norman GR. The value of basic science in clinical diagnosis: Creating coherence among signs and symptoms. Med Educ 2005; 39 (1): 107-12.

17. Torrealba C. Pioneros. El inicio de la Biología experimental en Chile. Ciencia y Vida; 2014. 336 p.

18. Engels F. Pharmacology education: Reflections and challenges. Eur J Pharmacol 2018; 833: 392-5.

19. Woodman O, Dodds A, Frauman A, Mosepele M. Teaching pharmacology to medical students in an integrated problem-based learning curriculum: an Australian perspective. Acta Pharmacol Sin 2016; 25 (9): 1195-203.

20. The Association of Faculties of Medicine of Canada (AFMC). The Future of Medical Education in Canada (FMEC): A Collective Vision for MD Education. 2010.

21. The Association of Faculties of Medicine of Canada (AFMC). FMEC MD 2015; Five Years of Innovations at Canadian Medical Schools. 2015.

22. Cumming A. The Bologna process, medical education, and integrated learning. Med Teach 2010; 32 (4): 316-8.

23. Two-Cycle Degree Structure in Medical Education in the EHEA [Internet]. [citado el 19 de noviembre de 2019]. Available from: https://wenr.wes.org/2018/12/two-cycle- degree-structure-in-medical-education-in-the-ehea

24. Zavlin D, Jubbal KT, Noé JG, Gansbacher B. A comparison of medical education in Germany and the United States: from applying to medical school to the beginnings of residency. Ger Med Sci 2017; 15: Doc15.

25. Pernas M, G Arancibia L, Gari M, Nogueira M, Rivera N. Ciencias básicas biomédicas y aprendizaje de la clínica en la formación de médicos en Cuba. Revista de Docencia Universitaria 2012; 10: 119-47.

26. Competencias comunes para los egresados de las Escuelas de Medicina ASOFAMECH [Internet]. 2016 [cited 2019 Oct 6]. Available from: http://www.carlosjorquera. com/CompetenciasPerfilASOFAMECHfinal.pdf

27. ASOFAMECH. Perfil de conocimientos EUNACOM [Internet]. 2010 [citado el 6 de octubre de 2019]. Available from: http://www.eunacom.cl/contenidos/PerfilNew. pdf

28. Brauer DG, Ferguson KJ. The integrated curriculum in medical education: AMEE Guide No. 96. Med Teach 2015; 37 (4): 312-22.

29. Bate E, Hommes J, Duvivier R, Taylor DCM. Problem-based learning (PBL): Getting the most out of your students-Their roles and responsibilities: AMEE Guide No. 84. Med Teach 2014; 36 (1): 1-12.

30. Concha M. ¿Es tiempo de modificar la vinculación pregrado postgrado en la educación médica chilena? Reflexiones sobre una vía de doble titulación médico cirujano/doctor en ciencias médicas. Rev Med Chile 2017; 145 (12): 1569-78.

31. Roberts SF, Fischhoff MA, Sakowski SA, Feldman EL. Perspective: transforming science into medicine: how clinician-scientists can build bridges across research's "valley of death". Academic Medicine 2012; 87 (3): 26670.

32. Labbe TP, Barake F, Alamos MF, Molina C, Ríos JA. Realidad de la Investigación en Salud en Chile: participación de los Médicos en el Fondo Nacional de Investigación (FONIS) en la última década. ARS MEDICA Rev Ciencias Médicas 2017; 42 (2): 76-80.

33. Salas S, Rigotti A. Médicos-Científicos en Chile: ¿Una especie en extinción? Rev Med Chile 2005; 133 (1): 1218.

34. Jain MK, Cheung VG, Utz PJ, Kobilka BK, Yamada T, Lefkowitz R. Saving the Endangered Physician-Scientist - A Plan for Accelerating Medical Breakthroughs. N Engl J Med 2019; 381 (5): 399-402.

35. Acuña MR, Ríos JA, Labbé TP. Hipótesis: Un paso central en el pensamiento clínico y la generación de evidencia científica. Rev Chil Radiol 2019; 25 (3): 83-6. 